\title{
Molecular detection of Extended-Spectrum $\beta$-lactamases (ESBLs) and biofilm formation in uropathogen Klebsiella pneumoniae in Iran
}

\author{
Elham Haghighifar ${ }^{1}$, Fatemeh Norouzi ${ }^{2}$, Razie Kamali Dolatabadi ${ }^{1} *$ (D) \\ Received: 14 Jun 2020 \\ Published: 5 Jun 2021
}

\section{Abstract}

Background: Uropathogenic Klebsiella pneumoniae is one of the well-kown uropathogens that have the main rule in biofilm formation. Increased prevalence of ESBL enzyme is one of the therapeutic problems. However, the aims of this study were to characterize the ability of biofilm formation and ESBL-producing isolates produced by urinary tract infection's K. pneumoniae to identify the prevalence of this type of infection in the studied area.

Methods: Between the 500 nonrepetitive clinical isolates, 128 isolates were detected as K. pneumoniae. Biofilm production of these isolates was showed by Merrit and Christensen method. The standard Kirby-Bauer disk diffusion method was used for antimicrobial susceptibility testing. The phenotype ESBL was confirmed by double disc synergy test (DDST). Genotypic identification of ESBLs did by molecular detection. The statistical analysis was done using software IBM SPSS Statistics (ISPSS Inc) and chi-square and Fisher exact tests.

Results: The result of microtiter plate was observed and it was found that $86(67.2 \%)$ isolates had weak biofilm, $24(18.8 \%)$ moderate biofilm, and $18(14.1 \%)$ strong biofilm. Also, 57 (44.5\%) out of 128 isolates were diagnosed as MDR. The highest frequency of resistance was identified for cefotaxime $60(46.9 \%)$ and tetracycline $60(46.9 \%)$, and the lowest rate was for amikacin $16(12.5 \%)$. The results of DDST showed 55 of 128 (43\%) produced ESBL enzymes. PCR detection in ESBL-producing isolates showed contained bla $_{T E M} 33$ of 55(63.1\%), and bla $a_{V E B} 13$ of 55 (23\%). Also, 1 of $55(2 \%)$ had both bla TEM $_{\text {and }}$ bla $a_{V E B}$. Also, 5 of 13 (38.4\%) isolates that had the bla $_{V E B}$ gene were also MDR and had weak biofilm $(8 / 13 ; 61.5 \%)$, intermediate biofilm $(3 / 13 ; 23 \%)$, and strong biofilm $(2 / 13 ; 15.4 \%)$.

Conclusion: To decrease treatment complications and mortality rate of drug-resistant bacterial infections, rapid detection of $\beta$ lactamases genes and evaluation of these properties and infection management programs can help to prevent the transmission of drug resistant-strains.

Keywords: Extended-spectrum $\beta$-lactamases (ESBLs), Biofilm Formation, Klebsiella pneumonia, Antibiotic resistance, bla $_{T E M}$, bla $_{V E B}$

Conflicts of Interest: None declared

Funding: None

\section{*This work has been published under CC BY-NC-SA 1.0 license. \\ Copyright $\odot$ Iran University of Medical Sciences}

Cite this article as: Haghighifar E, Norouzi F, Kamali Dolatabadi R. Molecular detection of Extended-Spectrum $\beta$-lactamases (ESBLs) and biofilm formation in uropathogen Klebsiella pneumoniae in Iran. Med J Islam Repub Iran. 2021 (5 Jun);35:72. https://doi.org/10.47176/mjiri.35.72

\section{Introduction}

Urinary tract infection (UTI) covers a wide range of asymptomatic microbial colonization to inflammation and widespread microbial invasion into the urinary tract (1). One of the main of UTI morbidity and mortality is the infection in the hospital as a nosocomial infection that is

Corresponding author: Razie Kamali Dolatabadi, r.kamali@resident.mui.ac.ir

1. Department of Microbiology, School of Medicine, Isfahan University of Medical Sciences, Isfahan, Iran

2. Department of Microbiology, School of Medicine, Fasa University of Medical Sciences, Fasa, Iran said to account for $25 \%$ to $40 \%$ of nosocomial infections (2).

Klebsiella pneumoniae is a gram-negative and facultative anaerobic bacteria that is capable of causing a wide range of health care-associated infections (3). Drug

$\uparrow$ What is "already known" in this topic:

Klebsiella pneumoniae is one of the well-kown uropathogens. The enzyme $\beta$-lactamase is the major cause of resistance to betalactam antibiotics in gram-negative bacteria.

$\rightarrow$ What this article adds:

The findings of this study could help select the appropriate antibiotic treatment. These findings suggest that genes involved in antibiotic resistance are increasing. 
resistance is a major problem in almost all countries. Antibiotic multidrug resistant (MDR) in K. pneumoniae is conferred primarily by extended spectrum $\beta$-lactamase (ESBL), which are enzymes that hydrolyze the $\beta$-lactam ring of $\beta$-lactam antibiotics (4).

Broad-spectrum beta-lactamase microbial resistance was first observed in early 1980s in Europe and then in the United States shortly after the entry of the third-generation cephalosporins into the treatment line (5). In gramnegative bacteria, the main reason for resistance to betalactam antibiotics is the enzyme $\beta$-lactamase. Today, there is an increase outbreak and dissemination of ESBL enzymes that hydrolyze and cause resistance to oxyiminocephalosporins and aztreonam (6). More than 300 different ESBL types are known. However, temoneira (TEM) and sulphydryl variable (SHV) types are widespread ESBLs and CTX-M ESBL is rapid spread (7). TEM, SHV, and OXA were predominant enzyme types until the late 1990s $(8,9)$. New types that have emerged today consist of families of pseudomonas extendedresistance (PER) and Vietnamese extended-spectrum beta-lactamase (VEB) (10). VEB and PER are rare species and their study is valuable. However, the virulence factors expression, such as genes involved in biofilm production and virulence, plays an significant role in treatment failure among infections caused by highly resistant $K$. pneumoniae isolates (11). Several virulence genes expressions have been related to the persistence and increased survival of $K$. pneumoniae within the host; they consist of quorum sensing genes (12) and genes involved in biofilm formation, which eventually lead to improvement of drug resistance $(13,14)$.

Biofilm formation is a transitional state of planktonic life, characterized by the adherent accumulation of small bacterial colonies surrounded by extracellular polysaccharide matrix, in which cells are irreversibly attached to various surfaces, including medical devices and damaged tissues (15).

The aims of this study were to characterize the ability of biofilm formation and ESBL-producing $K$. pneumoniae isolates and identify the types of ESBL produced by urinary tract infections $K$. pneumoniae from a large university hospital.

\section{Methods}

The 500 nonrepetitive clinical isolates were collected from a university hospital in Isfahan, Iran, from March 2017 to April 2018. Also, written informed consent forms were taken from either the patients or their authorized representatives. This study was approved by the research ethics committee of Isfahan University of Medical Sciences. A total of 128 isolates from urine samples were initially identified as $K$. pneumoniae using the standard IMVIC biochemical tests (indole, methyl red, VogesProskauer, citrate) and urease. Confirmation of isolates was done by molecular detection of the ureD gene (243 bp), which hydrolyzed the urea (5). K. pneumoniae ATCC 700603 was used as the positive control. The primers were used as listed below:

ure -D F: 5_-CCCGTTTTACCCGGAAGAAG - 3 ure -D R: 5 - GGAAAGAAGATGGCATCCTGC -3

\section{Biofilm Production (by microtitre plates)}

Biofilm production was performed by a modification of the procedure illustrated by Merrit et al (2005) and Christensen et al (1985) $(16,17)$. The isolates were grown in Brain Heart Infusion Broth supplemented with 2\% glucose and sucrose overnight at $37 \cdot \mathrm{C}^{\circ}$ for 24 hours. The cultures were diluted and the cell suspension was used to inoculate sterile flat-bottonned 96-well polystyrene microtitre plate at $37 \cdot \mathrm{C}^{\circ}$ for 48 hours. After 48 hours, the suspension was poured off and the wells were washed 3 times gently. The dried wells were stained with $0.1 \%$ crystal violet solution for 20 minutes. Then, the wells were washed 3 times and allowed to dry. A strong biofilm formation can be observed stained layer adhered to the inner wall of the microtitre plate wells.

\section{Antimicrobial Susceptibility Testing}

Antimicrobial susceptibility testing was done using the standard Kirby-Bauer disk diffusion procedure on the Mueller-Hinton agar (Merck). Antibiotic discs were used for the early detection of ESBLs production in $K$. pneumoniae isolates. The guidelines from the Clinical Laboratory Standards Institute (CLSI, 2018) were applied for performance and interpretation (18).

The concentrations of antimicrobial agents used on the Mueller-Hinton agar were as follows: imipenem (10 $\mu \mathrm{g})$, tetracycline $(30 \mu \mathrm{g})$, trimethoprim $(1.25 \mu \mathrm{g})$ sulfamethoxazole $(23.75 \mu \mathrm{g})$, ceftriaxone $(10 \mu \mathrm{g})$, cefotaxime $(10 \mu \mathrm{g})$, amikacin $(30 \mu \mathrm{g})$, ciprofloxacin $(5 \mu \mathrm{g})$, gentamicin $(10 \mu \mathrm{g})$, cefepime $(30 \mu \mathrm{g})$, and nitrofurantoin $(10 \mu \mathrm{g})$ (American Bidi). E. coli ATCC 25922 was used as a negative control (19).

\section{ESBLS Screening and Phenotypic Identification by DDST}

In early screening, detected resistant or decreased sensitivity to more than one of the third generation cephalosporins (3GC-ceftazidime, cefotaxime) were considered as probable ESBL producers (5). The phenotype identification was performed by Double Disc Synergy Test (DDST) on the Mueller-Hinton agar. Antibiotic discs of ceftazidime and ceftazidime-clavulanic acid were placed $15 \mathrm{~mm}$ apart and the plates were placed under aerobic incubation at $37^{\circ} \mathrm{C}$. An increase of more than $5 \mathrm{~mm}$ in zone diameter for either antimicrobial agent tested in combination with clavulanic acid over the initial zone diameter of the agent assayed was defined as ESBL (18). K. pneumonia ATCC700603 and E. coli ATCC 25922 were used as a positive and negative control, respectively.

\section{ESBLs Genotypic identification}

The $K$. pneumoniae DNA template was extracted from freshly cultured isolates by suspending colonies in Distilled water 2 times and boiling it for 10 minutes. The samples were cooled for 5 minutes at $-20^{\circ} \mathrm{C}$ and then centrifuged for 10 minutes at $10000 \mathrm{rpm}$ (9). The 
Table 1. Primers Used for Amplification of ESBLs Genes

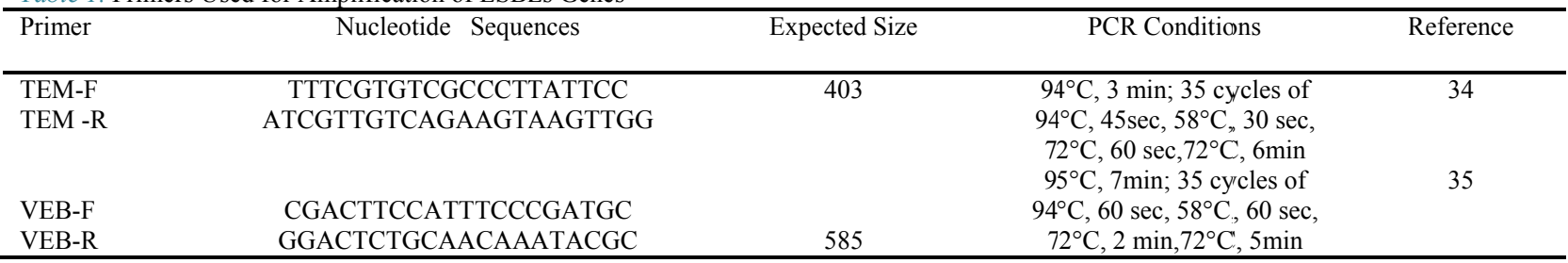

supernatant was applied as the DNA template in polymerase chain reaction (PCR) analysis. The isolates were identified as ESBL by molecular detection of bla $a_{\mathrm{TEM}}$, $b l a_{\mathrm{VEB}}$. The primers of these genes are listed in Table 1 . A PCR reaction tube was done in $30 \mu \mathrm{l}$ mixture of $3 \mu \mathrm{L} 10 \mathrm{X}$ buffer, $1 \mu \mathrm{L}$ of $10 \mathrm{mM} \mathrm{MgCl}_{2}$, and $0.25 \mu \mathrm{L}$ of $5 \mathrm{U} / \mu \mathrm{L}$ Taq DNA polymerase (Fermentas, Lithuania), $0.5 \mu \mathrm{L}$ each of $10 \mathrm{mM}$ deoxynucleaotidetriphosphates, $1 \mu \mathrm{L}$ each of 10 $\mu \mathrm{M}$ primers, and $5 \mu \mathrm{L}$ of plasmid extract in a thermal cycler (Kyratec).

\section{Data Analysis}

Data were analyzed via Statistical Package for the Social Sciences (SPSS) version 24 (SPSS Inc). The relation between biofilm production and the amount of biofilm production with drug resistance phenotypes of $K$. pneumoniae was determined by chi-square and Fisher exact tests. The analysis was detected with a confidence level of $95 \%$ and $\mathrm{p}<0.05$ was considered statistically significant.

\section{Results}

The 128 clinical isolates of $K$. pneumoniae were detected by biochemical tests. Molecular identification was performed by PCR of ure D gene and all isolates were positive. Among $128 \mathrm{~K}$. pneumonia clinical isolates, 76 $(59.3 \%)$ were from males and $52(40.6 \%)$ were from females. The result of microtiter plate was $128(100 \%)$ observing biofilm production. Also, $86(67.2 \%)$ isolates had weak biofilm, $24(18.8 \%)$ moderate biofilm, and 18 (14.1\%) strong biofilm (Table 2). In this study, MDR profile was considered resistance to at least 3 classes of antimicrobial agents. A total of 57 isolates (44.5\%) were identified as MDR.

The Pattern of Resistance to Antimicrobial agents is shown in Figure 1.

Our results indicated that $(55 / 128 ; 43 \%) \mathrm{K}$. pneumoniae isolates were producing ESBL. Our results indicated the highest frequency of resistance was demonstrated for cefotaxime $60(46.9 \%)$ and etracycline $60(46.9 \%)$ and the lowest rate was for amikacin $16(12.5 \%)$. We further surveyed the correlation of the MDR profile of the isolates with the ability to form biofilm. Comparison of the drug resistance profile with their biofilm strength index revealed that in MDR isolates, 37 (65\%) were weak and $12(21 \%)$ were moderate, while the strong biofilm formers were only $8(14 \%)$. In statistical studies, it was observed that the strains that were resistant to ciprofloxacin and tetracycline were significantly correlated with biofilm production, with a probability value of 0.037 and 0.021 .
The results of DDST of $K$. pmeumoniae isolates showed that 55 of $128(43 \%)$ isolates produced ESBL enzymes. Molecular detection showed that ESBL-producing $K$. pneumoniae isolates had $63.1 \%$ bla $a_{\text {TEM }}(35 / 55)$ and $23 \%$ bla $_{\mathrm{VEB}}(13 / 55)$; also, 1 of $55(2 \%)$ had both $b l a_{\mathrm{TEM}}$ and $b l a_{\mathrm{VEB}}$ (Fig. 2). Moreover, 5 of 13 (38.4\%) isolates that

\begin{tabular}{lcc} 
Table 2. Biofilm Formation in K. pneumonia & \\
\hline Biofilm & Frequency & $\%$ \\
\hline Weak & 86 & 67.2 \\
Intemediate & 24 & 18.8 \\
Strong & 18 & 14.1 \\
Total & 128 & 100 \\
\hline
\end{tabular}

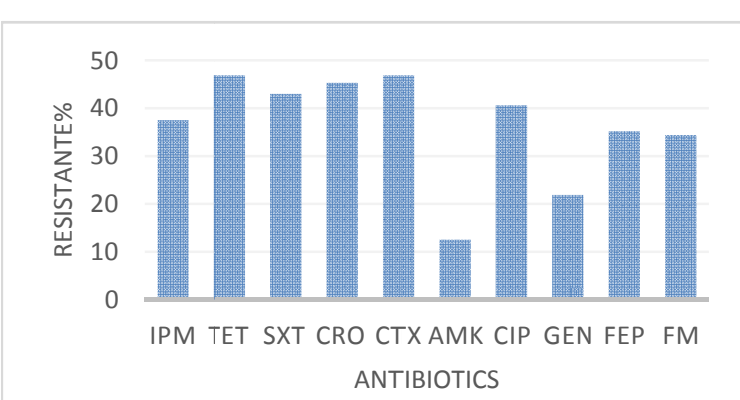

Fig. 1. Pattern of Resistance to Antimicrobial Agents Among all K. pneumonia Strains

a Abbreviations: IPM, Imipenem; TET, Tetracycline; SXT, Trimethoprim/sulfamethoxazole , Ceftriaxone,CRO; Cefotaxime, CTX; Amikacin,AMK; Ciprofloxacin,CIP; Gentamiicin,GEN; Cefepime,FEP; Nitrofurantoin, FM.

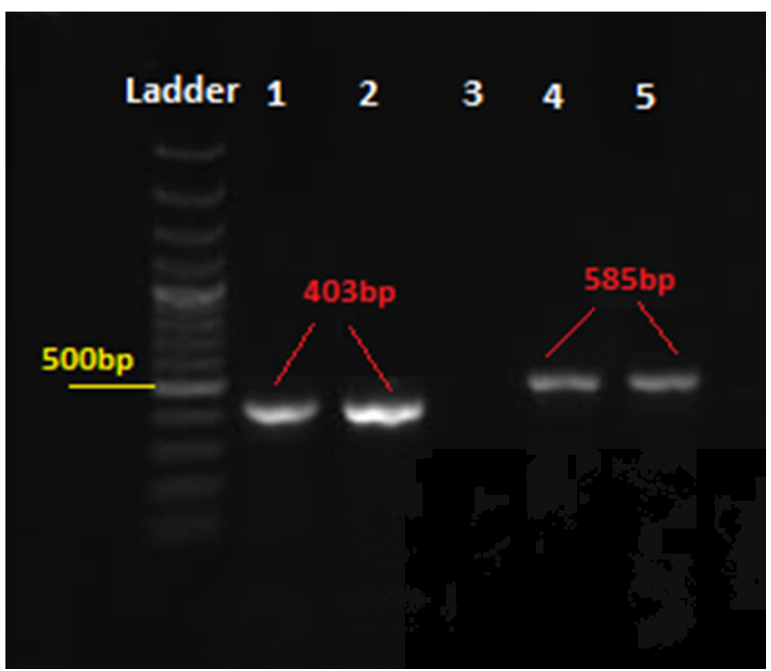

Fig. 2. Polymerase Chain reaction Amplification of bla $a_{\mathrm{VEB}}$ and bla ${ }_{\text {TEM. }}$.

Lanes 1: positive control bla $\mathrm{a}_{\mathrm{TEM}}$, Lanes 2: PCR product of bla $\mathrm{a}_{\mathrm{TEM}}(403 \mathrm{bp})$ Lane 3: Negative control, Lane 4: positive control bla $\mathrm{V}_{\mathrm{VEB}}$, Lanes 5: PCR product of bla VEB $_{\text {(n) }}(585 \mathrm{bp})$. 
had the $b l a_{\text {VEB }}$ gene were also MDR with weak biofilm $(8 / 13 ; 61.5 \%)$, intermediate biofilm $(3 / 13 ; 23 \%)$, and strong biofilm $(2 / 13 ; 15.4 \%)$. Also, 14 of $33(42.4 \%)$ isolates that had the $b l a_{\text {TEM }}$ gene were also MDR withweak biofilm $(24 / 33 ; 72.7 \%)$, intermediate biofilm $(5 / 33 ; 15 \%)$, and strong biofilm $(4 / 33 ; 12.12 \%)$. There was a significant relationship between the presence of $b l a_{\mathrm{VEB}}$ gene and cefepime resistance. Also, based on the Pearson chi-square test results, there was an important association between the presence of $b l a_{\mathrm{TEM}}$ gene and resistance to ceftriaxone and cefotaxime.

\section{Discussion}

Recently $K$. pneumoniae resistance to beta-lactam antibiotics has become a worldwide problem in the field of health care. Increased multidrug-resistant $K$. pneumoniae as a serious nosocomial pathogen in patients with urinary tract infection is an important and significant issue. Nowadays, removing MDR UTI is very challenging because not only the hard treatmenting strains are growing but also the antimicrobial resistance is developing (21, 22). Therefore, recognition of the contribution of those strains in infectivity hospitalized patients, in addition to continuous tracking of involved genes in each region, can significantly contribute to the prevention and control of these infections (23). We additionally present document proposed that the MDR profile of K.pneumoniae is not related to the biofilm strength. In our study, the highest frequency of resistance was to cefotaxime $(46.9 \%)$ and tetracycline $(46.9 \%)$ that was lower than those reported from other Iranian cities by Azizian et al from Hamedan in 2019 (50\%), Eghbalpoor et al from Tehran in 2019 (63.3\%), Sara Kootia et al from Shiraz in 2019 (50\%), and Jahromy et al from Tehran in 2019 (100\%) (24-26). In this study, the most effective antibiotic was amikacine with $87.5 \%$ sensitivity that is different from other studies, including those of Hossein Forouzandeh (2019) (71.6\%) and SajjadYazdansetad (2019) (north-central Iran) (50\%). This may be due to the optimal management of infection control at the hospital under study in this study $(27,28)$. Many bacterial chronic infections due to biofilm formation are difficult to deal with and remove. Biofilm production in $K$. pneumoniae increases colonization and leads to increased UTI. Treatment of these infections is difficult because they render multiple drug resistance (29). In the current study, $78 \%$ of isolates were capable of forming biofilms, while in another study in patients with catheter-associated UTI, $89.5 \%$ of isolates produced biofilm and among those patients without catheterassociated UTI none produced biofilm (30). In this study, we did not find an association between drug resistance profile and biofilm formation ability, although DiagoNavarro et al reported a correlation within the $K$. pneumoniae isolates causing blood infections (31). In the present study, the strains were resistant to ciprofloxacin, and tetracycline was significantly correlated with biofilm by production, with a probability value of 0.037 and 0.021 . The cause of this phenomenon in a Caenorhabditis elegans model was shown by Bialek et al between overexpression of efflux pump and increasing virulence
(32). Our result to detect the prevalence of several $\beta$ lactamase in $K$. pneumoniae clinical isolates revealed that there were $43 \% \mathrm{~K}$. pneumoniae isolates. In our study, $43 \%$ of $K$. pneumoniae isolates produced ESBL enzymes, $63.1 \% b l a_{\mathrm{TEM}}, \quad 23 \% \quad b l a_{\mathrm{VEB}}$, and $2 \% \quad b l a_{\mathrm{TEM}}$ and $b l a_{\mathrm{VEB}}$. While Tahanasab $\mathrm{Z}$, et al reported that $60.4 \%$ isolates were ESBL-producers and $81 \%$ carried the $b l a_{\text {TEM }}$ gene (33). Sedighi M, et al reported that the prevalence of $b l a_{\mathrm{TEM}}$ and $b l a_{\mathrm{VEB}}$ was $38 \%$ and $6 \%$, respectively (34). Bora et al from India showed frequency of $3 \beta$-lactamase genes ( $b l a_{\mathrm{TEM}}, b l a_{\mathrm{VEB}}$ and $\left.b l a_{\mathrm{CTX}-\mathrm{M}}\right)$ but found that $b l a_{\mathrm{TEM}}$ was predominant in $K$. pneumoniae producing $\beta$ lactamase (ESBL positive isolates) (77.58\%) (35). However, in Asia as well as in this study, bla $a_{\mathrm{TEM}}$ has become the predominant type of $\beta$-lactamase types in $K$. pneumoniae clinical isolates (36).

\section{Conclusion}

This study demonstrated the increased considerable bla $a_{\mathrm{TEM}}$ and $b l a_{\mathrm{VEB}}$ prevalence of $K$. pneumoniae strains circulating in hospitals in Isfahan, Iran. This MDR incremental trend is correlated with the presence of $b l a_{\text {TEM. }}$. The high prevalence of drug resistance genes indicates the necessity for serious monitoring of antibiotic administration to control infections caused by Enterobacteriaceae in Iran. However, to decrease complications of treatment and mortality rate for drugresistant bacterial infections, rapid diagnosis of $\beta$ lactamases genes and evaluation of these properties could help to prevent transmission of drug resistant-strains and infection management programs. Also, further studies are required to get gather more detailed and impressive results.

\section{Acknowledgement}

We thank all the staff at the Microbiology Department of the Isfahan University of Medical Sciences.

\section{Conflict of Interests}

The authors declare that they have no competing interests.

\section{References}

1. Amadu DO, Nwabuisi C, Usman Y, Mustapha JO, Abdullahi IN Popoola AA. Biofilm and Extended Spectrum Beta Lactamase Production amongst Uropathogenic Escherichia Coli Isolates at the University of Ilorin Teaching Hospital, Nigeria Int J MedLab. 2019 Dec 14.

2. Aboderin OA, Abdu AR, Odetoyin BW, Lamikanra A. Antimicrobial resistance in Escherichia coli strains from urinary tract infections. J Natl Med Assoc. 2009 Dec 1;101(12):1268-73.

3. Gheitani L, Fazeli H. Prevalence off bla VIM, bla IMP, and bla KPC Genes among Carbapenem-Resistant Klebsiella pneumoniae (CRKP) Isolated from Kurdistan and Isfahan Hospitals. Iran Res Mol Med. 2018 May 10;6(2):12-20.

4. Asensio A, Oliver A, González-Diego P, Baquero F, Perez-Diaz JC, Ros P, et al. Outbreak of a multiresistant Klebsiella pneumoniae strain in an intensive care unit: antibiotic use as risk factor for colonization and infection. Clin Infect Dis. 2000 Jan 1;30(1):55-60.

5. Fazeli H, Kamali Dolatabadi R, Taraghian A, Nasr Isfahani B, Moghim S. Genetic Characterization of blaSHV/VEB/PER Genes in ESBL-producing MDR Klebsiella Pneumonia Strains Isolated from Patients in Isfahan, Iran. Eur Online J Nat Soc Sci. 2015 Mar 30;4(1):191. 
6. Ahmed OI, El-Hady SA, Ahmed TM, Ahmed IZ. Detection of bla SHV and bla CTX-M genes in ESBL producing Klebsiella pneumoniae isolated from Egyptian patients with suspected nosocomial infections. Egypt J Med Hum Genet. 2013;14(3):277-83.

7. Al Jasser AM. Extended-spectrum beta-lactamase (ESBLS): a global problem.

8. Al-Zarouni M, Senok A, Rashid F, Al-Jesmi SM, Panigrahi D. Prevalence and antimicrobial susceptibility pattern of extendedspectrum beta-lactamase-producing Enterobacteriaceae in the United Arab Emirates. Med Princ Pract2008;17(1):32-6.[ doi:10.1159/000109587]

9. Amjad A, Mirza IA, Abbasi SA, Farwa U, Malik N, Zia F. Modified Hodge test: A simple and effective test for detection of carbapenemase production. Iran J Microbiol. 2011 Dec;3(4):189.

10. Jog AS, Shadija PG, Ghosh SJ. Detection of multidrug resistant Gram-negative bacilli in type II diabetic foot infection. Int J Med Health Sci. 2013 Apr;2:186-94.

11. Bahador A, Farshadzadeh Z, Raoofian R, Mokhtaran M, Pourakbari B, Pourhajibagher M, Hashemi FB. Association of virulence gene expression with colistin-resistance in Acinetobacter baumannii: analysis of genotype, antimicrobial susceptibility, and biofilm formation. Ann Clin Microbiol Antimicrob2018 Dec 1;17(1):24.[ doi:10.1186/s12941-018-0277-6]

12. Zarrilli R. Acinetobacter baumannii virulence determinants involved in biofilm growth and adherence to host epithelial cells. VIRULENCE. $2016 \quad$ May 18;7(4):367-8. [ doi:10.1080/21505594.2016.1150405]

13. Lee J, Page R, García-Contreras R, Palermino JM, Zhang XS, Doshi O, Wood TK, Peti W. Structure and function of the Escherichia coli protein YmgB: a protein critical for biofilm formation and acidresistance. J. Mol. Biol. 2007 Oct 12;373(1):11-26.[ doi.org/10.1016/j.jmb.2007.07.037]

14. Wroblewska MM, Sawicka-Grzelak A, Marchel H, Luczak M, Sivan A. Biofilm production by clinical strains of Acinetobacter baumannii isolated frompatients hospitalized in two tertiary care hospitals. FEMS IMMUNOL MED MIC. 2008 Jun 1;53(1):140-4.[ doi.org/10.1111/j.1574-695X.2008.00403.x]

15. Hassan A, Usman J, Kaleem F, Omair M, Khalid A, Iqbal M. Evaluation of different detection methods of biofilm formation in the clinical isolates Braz J Infect Dis. 2011 Aug;15(4):305-11.

16. Merritt JH, Kadouri DE, O'Toole GA. Growing and analyzing static biofilms. Current protocols in microbiology. 2011 Aug;22(1):1B.[ doi:10.1002/9780471729259.mc01b01s22]

17. Christensen GD, Simpson WA, Younger JJ, Baddour LM, Barrett FF, Melton DM, Beachey EH. Adherence of coagulase-negative staphylococci to plastic tissue culture plates: a quantitative model for the adherence of staphylococci to medical devices. J CLIN MICROBIOL. 1985 Dec 1;22(6):996-1006.

18. CLSI. M100 Performance Standards for Antimicrobial.

19. Fazeli H, Kamali Dolatabadi R, Taraghian A, Nasr Isfahani B, Moghim S. Genetic Characterization of blaSHV/VEB/PER Genes in ESBL-producing MDR Klebsiella Pneumonia Strains Isolated from Patients in Isfahan, Iran. Eur. Online J. Nat. 2015 Mar 30; 4(1): pp191.

20. Kumar VA, Khan S. Defining multidrug resistance in Gram-negative bacilli. Indian Journal of Medical Research. 2015 Apr 1;141(4):491.

21. Doi Y, Murray GL, Peleg AY. Acinetobacter baumannii: evolution of antimicrobial resistance - treatment options. InSeminars in respiratory and critical care medicine 2015 Feb (Vol. 36, No. 01, pp. 085-098). Thieme Medical Publishers.[ doi: 10.1055/s-0034-1398388]

22. Potron A, Poirel L, Nordmann P. Emerging broad-spectrum resistance in Pseudomonas aeruginosa and Acinetobacter baumannii: mechanisms and epidemiology. Int J Antimicrob Agents. 2015 Jun 1;45(6):568-85.[ doi:10.1016/j.ijantimicag.2015.03.001]

23. Rezaei A, Fazeli H, Moghadampour M, Halaji M, Faghri J. Determination of antibiotic resistance pattern and prevalence of OXAtype carbapenemases among Acinetobacter baumannii clinical isolates from inpatients in Isfahan, central Iran. Infez Med. 2018 Mar 1;26(1):61-6.

24. Azizian K, Alikhani MY, Mashouf RY, Gholizadeh P, Noshak MA. Antibiotic resistance patterns and frequency of metallo-betalactamases in Klebsiella pneumoniae in Hamadan, Iran. Journal of Contemporary Clinical Practice.. 2019 May 1;5(1):10-7.

25. Eghbalpoor F, Habibi M, Azizi O, Asadi Karam MR, Bouzari S. Antibiotic resistance, virulence and genetic diversity of Klebsiella pneumoniae in community-and hospital-acquired urinary tract infections in Iran. ACTA MICROBIOL IMM H. 2019 Sep;66(3):34966.

26. Jahromy SH, Rahbar M, Bialvaei AZ, Asadi A, Jahromy SH, Eslami P, Mardani M, Kouhsari E. Prevalence of fosfomycin resistance genes and antimicrobial susceptibility of clinical urinary extended-spectrum beta-lactamase-producing Escherichia coli and Klebsiella pneumoniae isolates. Rev Med Microbiol. 2019 Nov 4;30.

27. Piruozi A, Forouzandeh H, Farahani A, Forouzandeh Z, Ahmadi I, Abdizadeh R, et al. Investigating the Frequency of Klebsiella Infection and Drug Resistance Among Inpatients and Outpatients Referring to Amir Al-Momenin Hospital, Gerash, Iran. Gene Tissue Cell. 2019;6(3).

28. Yazdansetad S, Alkhudhairy MK, Najafpour R, Farajtabrizi E, AlMosawi RM, Saki M, et al. Preliminary survey of extended-spectrum $\beta$-lactamases (ESBLs) in nosocomial uropathogen Klebsiella pneumoniae in north-central Iran. Heliyon. 2019 Sep 1;5(9):e02349.

29. Cusumano JA, Caffrey AR, Daffinee KE, Luther MK, Lopes V, LaPlante KL. Weak biofilm formation among carbapenem-resistant Klebsiella pneumoniae. Diagn Microbiol Infect Dis. 2019 Dec 1;95(4):114877.

30. Karigoudar RM, Karigoudar MH, Wavare SM, Mangalgi SS, Detection of biofilm among uropathogenic Escherichia coli and its correlation with antibiotic resistance pattern. J Lab Physic. 2019 Jan;11(1):17.

31. Vuotto C, Longo F, Pascolini C, Donelli G, Balice MP, Libori MF, et al. Biofilm formation and antibiotic resistance in Klebsiella pneumoniae urinary strains. J Appl Microbiol. 2017 Oct;123(4):100318.

32. Bialek S, Lavigne JP, Chevalier J, Marcon E, Leflon-Guibout V, Davin A, et al. Membrane efflux and influx modulate both multidrug resistance and virulence of Klebsiella pneumoniae in a Caenorhabditis elegans model. Antimicrob Agents Chemother. 2010 Oct 1;54(10):4373-8.

33. Tahanasab Z, Mobasherizadeh S, Moghadampour M, Rezaei A, Maleki N, Faghri J. High Prevalence of Multiple Drug Resistance among ESBLs-Producing Klebsiella pneumoniae Isolated from Hospitalized Patients in Isfahan, Iran. J Med Bacteriol. 2016;5(56):29-38.

34. Sedighi M, Halajzadeh M, Ramazanzadeh R, Amirmozafari N, Heidary M, Pirouzi S. Molecular detection of $\beta$-lactamase and integron genes in clinical strains of Klebsiella pneumoniae by multiplex polymerase chain reaction. Rev Soc Bras Med Tro. 2017 Jun;50(3):321-8.

35. Bora A, Hazarika NK, Shukla SK, Prasad KN, Sarma JB, Ahmed G. Prevalence of blaTEM, blaSHV and blaCTX-M genes in clinical isolates of Escherichia coli and Klebsiella pneumoniae from Northeast India. Indian J Pathol Microbiol. 2014 Apr 1;57(2):249.

36. Bali EB, Acik L, Sultan N. Phenotypic and molecular characterization of SHV, TEM, CTX-M and extended-spectrum betalactamase produced by Escherichia coli, Acinobacter baumannii and Klebsiella isolates in a Turkish hospital. Afr J Microbiol Res. 2010 Apr 18;4(8):650-4. 